\title{
Effect of hospitalization on 24-h ambulatory blood pressure of hypertensive patients
}

\author{
Maria I Pikilidou${ }^{1}$, Efrosyni Tsirou${ }^{1}$, George S Stergiou ${ }^{2}$, Anastasios G Konstas ${ }^{3}$, Pantelis A Sarafidis ${ }^{1}$, \\ Anastasia Ptinopoulou ${ }^{1}$, Lina S Hadjistavri ${ }^{1}$, Panagiotis Georgianos ${ }^{1}$, Dimitrios G Mikropoulos ${ }^{3}$ \\ and Anastasios N Lasaridis ${ }^{1}$
}

The aim of this study is to assess the effect of hospital admission on 24-h ambulatory blood pressure (ABP) in hypertensive subjects. Treated or untreated hypertensive adults with open-angle glaucoma underwent inpatient and outpatient 24-h ABP monitoring in a random order 4 weeks apart. Awake ambulatory hours, awake in-bed hours and sleep hours were reported by participants. The nighttime-to-daytime ABP dip (\%) and the sleeping-to-awake dip (ambulatory and in-bed) were determined using the two ABP recordings. A total of 40 subjects were analyzed (mean age $65.7 \pm 8.4$ (s.d.) years, $n=19$ men). Daytime systolic BP (SBP) was lower in the hospital than in the outpatient setting (mean difference $4.3 \pm 10.4 \mathrm{~mm} \mathrm{Hg}, P=0.01$ ), as was the awake ambulatory SBP (mean difference $5.0 \pm 11.1 \mathrm{~mm} \mathrm{Hg}, P=0.008$ ). No differences were detected in $24 \mathrm{~h}$, nighttime or sleeping SBP or in any of the respective diastolic outpatient vs. inpatient ABP measurements. The nighttime SBP dip (vs. daytime) was larger in the outpatient setting $(8.9 \pm 7.5 \%$ and $5.2 \pm 4.7 \%$, respectively; $P=0.003$ ). Sleeping SBP dip (vs. awake ambulatory and awake in-bed) was also larger in the outpatient setting $(11.1 \pm 7.3$ and $7.8 \pm 5.9 \%$, respectively; $P=0.02)$ with no difference in diastolic ABP. These data suggest that inpatient 24-h ABP monitoring does not reflect the usual BP level during routine daily life, nor does it represent the usual diurnal pattern of an individual. Relying on the 24-h ABP monitoring performed in the hospital environment may lead to an underestimation of ABP and an overdiagnosis of non-dippers. Therefore, 24-h ABP monitoring for decision making regarding diagnosis and treatment of hypertension should be performed only in the routine daily conditions of each individual.

Hypertension Research (2010) 33, 995-999; doi:10.1038/hr.2010.127; published online 22 July 2010

Keywords: ambulatory blood pressure; inpatient blood pressure; nocturnal dip; sleeping dip

\section{INTRODUCTION}

Hypertension is a highly prevalent and modifiable risk factor of cardiovascular morbidity and mortality., ${ }^{1,2}$ Office blood pressure (BP) readings have been used in numerous longitudinal studies that have established the risks associated with elevated $\mathrm{BP}$; however, several lines of evidence clearly show that $24-\mathrm{h} \mathrm{BP}$ is more reproducible ${ }^{3}$ and superior to office measurements in predicting cardiovascular events and mortality, ${ }^{4,5}$ as well as in evaluating the true efficacy of antihypertensive drugs. ${ }^{6}$

Although it is recommended that 24-h ambulatory BP (ABP) be assessed during a period of routine daily activities, there are several circumstances in which this measurement is conducted in the hospital setting; for example, in cases of hospitalization for the diagnostic evaluation of hypertension and in patients hospitalized for other reasons.

However, the precise impact of hospitalization on an individual's BP levels and variability has not been thoroughly investigated.
Previous studies have shown a varied effect of hospitalization on $\mathrm{ABP}^{7-9}$ This effect could cause a decrease in BP as a result of factors such as the marked decrease in physical activity, limited exposure to behavioral stress or the placebo effect of non-antihypertensive medication. ${ }^{9}$ On the other hand, hospitalization could be a reason for an increase in BP due to the anxiety and stress induced by the hospital environment, the fear or possibility of being diagnosed with a serious disease and the negative impact of an unknown environment. ${ }^{8,10}$ Furthermore, hospital conditions may compromise sleep, affecting both sleep duration and quality and, consequently, the normal nocturnal BP dip.

Available data regarding the specific impact of hospitalization on 24-h BP measurements are scarce and contradictory. In addition, there are no available data on differences in the effect of the duration of sleep on BP levels, or on differences in BP dipping between the two environments. Therefore, the aim of the present study was to delineate the impact of hospitalization on 24-h ABP parameters in patients with

\footnotetext{
${ }^{1}$ Hypertension Center, 1st Department of Internal Medicine, Aristotle University of Thessaloniki, AHEPA Hospital, Thessaloniki, Greece; ${ }^{2}$ Hypertension Center, 3rd Department of Medicine, University of Athens, Sotiria Hospital, Greece and ${ }^{3}$ Glaucoma Unit, 1st University Department of Ophthalmology, AHEPA Hospital, Aristotle University, Thessaloniki, Greece

Correspondence: Dr MI Pikilidou, 1st Department of Internal Medicine, Aristotle University of Thessaloniki, AHEPA Hospital, St Kiriakidi 1, Thessaloniki 54636, Greece. E-mail: m_pikilidou@yahoo.com

Received 25 February 2010; revised 27 April 2010; accepted 10 May 2010; published online 22 July 2010
} 
mild untreated and treated hypertension, and also to assess differences in BP between the awake and sleeping hours and in the inpatient $v s$. outpatient settings.

\section{METHODS}

\section{Participants}

In all, 44 adults with newly diagnosed stage 1 hypertension or treated hypertension, who also had untreated ocular hypertension, were recruited from the glaucoma clinic of a university hospital. Exclusion criteria included secondary hypertension, stage 2 and 3 hypertensions, renal disease, sleep apnea, diabetes mellitus, acute or chronic inflammation, myocardial infarction or unstable angina within the past 6 months, heart failure (New York Heart Association class III and IV), active liver disease, history of drug or alcohol abuse or any other condition with poor prognosis. Smokers and subjects receiving treatment with non-steroidal anti-inflammatory agents, corticosteroids, $\beta$-blockers, or any other regimen that could affect BP levels were also excluded. None of the subjects received intravenous fluids during the in-hospital ABP monitoring. The protocol was approved by the Aristotle University of Thessaloniki Ethics Committee, and all participants signed an informed consent form. All examinations were conducted in accordance with the Declaration of Helsinki (1989 amendment).

\section{Protocol}

Patients underwent 24-h ABP monitoring twice-once when admitted in the hospital and once in the outpatient setting on a usual workday—in a random order 2-4 weeks apart. Randomization was performed by computer-generated random numbers to allocate the first monitoring setting in consecutive patients who met the eligibility criteria. For the in-hospital measurement, patients were admitted in the morning to the glaucoma unit of a university hospital and discharged the following day. During admission, they underwent measurement of their intraocular pressure at 1000, 1400, 1800, 2200 and 0000 hours. There was no change in antihypertensive treatment or glaucoma treatment throughout the study period. Breakfast, lunch and dinner, which corresponded to approximately $1500 \mathrm{kcal}$ daily and contained normal amounts of sodium, were given to each patient during hospitalization.

Participants were instructed to report the hours of their ambulatory, bed rest and sleeping hours during 24-h ABP monitoring in a diary. Sleep duration during $\mathrm{ABP}$ monitoring was self-evaluated by the participants in comparison with their regular habits and was classified as (a) normal, (b) $2 \mathrm{~h}$ less, (c) 2-4h less or (d) $>4 \mathrm{~h}$ less than normal. Furthermore, the quality of sleep during 24-h ABP monitoring was reported as (a) poor, (b) medium or (c) good. Mean systolic BP (SBP) and diastolic BP (DBP) were calculated for daytime, nighttime and the overall 24-h period. Mean SBP, DBP and heart rate during bed rest, sleeping hours and ambulatory hours were also calculated according to their activity questionnaires.

\section{Office and $\mathrm{ABP}$}

Office BP measurement was conducted according to the international guidelines. ${ }^{1,2}$ Measurements were taken by trained physicians using a standard mercury sphygmomanometer with a bladder size of $12 \times 35 \mathrm{~cm}$. The subject remained at rest in the sitting posture for at least $5 \mathrm{~min}$, and then the BP was measured. The diagnosis of hypertension was on the basis of three measurements of BP with 5 min between each measurement for two consecutive visits under the same conditions. The mean time between the two visits was 1 week. Korotkoff sounds I and V were used to define SBP and DBP, respectively. To establish a diagnosis of hypertension, the threshold of $140 / 90 \mathrm{~mm} \mathrm{Hg}$ for office BP was used. ${ }^{11}$

ABP was monitored using validated oscillometric Spacelabs 90217 devices (Spacelabs Medical, Redmond, WA, USA). ${ }^{12}$ The monitors, with a cuff of appropriate size, were fitted and ABP was recorded at 20-min intervals for $25 \mathrm{~h}$. The first hour of readings were not included in the analysis, as this period was used to enable participants to get used to the equipment function. Recordings were used in the analysis only if $\geqslant 80 \%$ of readings were valid, there were no more than 2 non-consecutive day hours (0800-2200 hours) with less than two valid readings and there was no more than 1 night hour (2200-0800 hours) without a valid reading. ${ }^{4,11}$ For daytime measurements, the $135 / 85 \mathrm{~mm} \mathrm{Hg}$ threshold for hypertension diagnosis was used. For nighttime, the 120/ $70 \mathrm{~mm} \mathrm{Hg}$ threshold was applied. ${ }^{7}$ The nocturnal BP dip (\%) was calculated as $100 \times(1-$ nighttime/daytime BP ratio), and the mean asleep SBP dip (\%) was calculated as $100 \times(1-$ sleep/awake BP). Patients with a nocturnal BP dip $<10 \%$ were classified as non-dippers, those with $10-20 \%$ were classified as dippers, those with $>20 \%$ nocturnal dip were classified as extreme dippers and those with BP higher during nighttime than during daytime were classified as reverse dippers.

\section{Analysis}

It was estimated that by including 40 subjects, the study would have $80 \%$ power to detect a difference of $5 \mathrm{~mm} \mathrm{Hg}$ in 24-h SBP between inpatient and outpatient measurements assuming a s.d. of $8 \mathrm{~mm} \mathrm{Hg}$ for a two-sided test and an $\alpha=0.05$. Student's $t$-test, Mann-Whitney $\mathrm{U}$ and $\chi^{2}$-tests were used when appropriate. Pearson's correlations were used to assess both the relationship between the measurements in the two settings and the s.d. of BP dispersion.

Multiple regression models were conducted. The dependent variable was the difference in ABP parameters between the inpatient and the outpatient setting, and the independent variables were age, gender, body mass index and antihypertensive therapy (yes/no).

Bland-Altman plots were used to show the limits of agreement between ABP parameters that differed significantly in the two settings. The coefficient of variation between the two measurements was also calculated according to the following formula: $\mathrm{CV}=($ s.d. $/ \sqrt{ } 2) \quad \bar{x} 100 / \bar{x}$ (s.d., difference between in-hospital and outpatient measurement; $\bar{x}$, pooled mean value for the two measurements). Variables were expressed as means \pm s.d. if they were normally distributed, or as medians with ranges if not. Discrete variables were reported as counts. Study power estimation was performed using NQuery Advisor software (Statistical Solutions, version 5.0, Boston, MA, USA), and statistical analyses were calculated using SPSS software (SPSS, version 13.0, Chicago, IL, USA).

\section{RESULTS}

A total of 44 subjects were recruited, 4 of which were lost to follow-up and excluded. In all, 40 subjects were analyzed (mean age $65.7 \pm 8.4$ (s.d.) years, 19 men); 18 were newly diagnosed hypertensive patients and the others had been previously diagnosed. Table 1 shows the basic demographic and clinical characteristics of the study population.

A comparison of ABP parameters obtained in the outpatient $v s$. the in-hospital setting is presented in Table 2. Daytime SBP was higher in the outpatient setting, which was mainly due to higher SBP during daytime

Table 1 Basic demographic and clinical characteristics of the study population

Values

Gender (male/female) (19/21)

Age (years) $\quad 65.7 \pm 8.4$

Glucose (mg/dl)

$98.1 \pm 9.6$

Urea (mg/dl)

$39.9 \pm 10.8$

Creatinine $(\mathrm{mg} / \mathrm{dl})$

$0.94 \pm 0.2$

Uric acid $(\mathrm{mg} / \mathrm{dl}) \quad 5.4 \pm 1.4$

$\mathrm{K}^{+}(\mathrm{mEq} / \mathrm{l})$

$\mathrm{Na}^{+}(\mathrm{mEq} / \mathrm{l})$

$4.2 \pm 0.45$

$\mathrm{Ca}^{2+}(\mathrm{mg} / \mathrm{dl})$

$140.6 \pm 3.1$

SGOT (IU/I)

$9.2 \pm 0.4$

SGPT (IU/I)

$25.1 \pm 18.7$

Cholesterol (mg/dl)

$25.1 \pm 20.8$

Triglycerides (mg/dl)

$209.6 \pm 43.3$

$\mathrm{HDL}(\mathrm{mg} / \mathrm{dl})$

$118.8 \pm 62.0$

$\mathrm{A} / \mathrm{C}(\mathrm{g} / \mathrm{g})$

$54.4 \pm 14.4$

8.5 (58)

Abbreviations: $A / C$, albumin to creatinine ratio; $H D L$, high-density lipoprotein All values are mean \pm s.d., except gender, which is in absolute numbers and $A / C$ in median (range). 
Table 2 Comparison of ambulatory blood pressure values during in-hospital and outpatient monitoring

\begin{tabular}{|c|c|c|c|c|c|c|c|}
\hline & $\begin{array}{c}\text { Outpatient } \\
\text { (mean } \pm \text { s.d.) }\end{array}$ & $\begin{array}{l}\text { In-hospital } \\
\text { (mean } \pm \text { s.d.) }\end{array}$ & $\begin{array}{c}\text { Difference } \\
\text { (mean } \pm \text { s.d.) }\end{array}$ & $\begin{array}{c}\text { 95\% Confidence } \\
\text { intervals }\end{array}$ & P-value & $\begin{array}{l}\text { Correlation } \\
\text { coefficient, r }\end{array}$ & $\begin{array}{l}\text { Coefficient of } \\
\text { variation (\%) }\end{array}$ \\
\hline 24-h SBP & $125.7 \pm 12.1$ & $122.9 \pm 11.7$ & $2.5 \pm 10.8$ & -0.9 to 6.0 & NS & 0.58 & 8.8 \\
\hline 24-h DBP & $73.6 \pm 9.0$ & $72.8 \pm 8.9$ & $0.6 \pm 5.0$ & -2.2 to 0.9 & NS & 0.56 & 6.9 \\
\hline 24-h HR & $66.0 \pm 8.1$ & $65.3 \pm 8.7$ & $-0.4 \pm 4.8$ & -1.9 to 1.2 & NS & 0.44 & 6.7 \\
\hline Daytime SBP & $129.0 \pm 12.2$ & $125.2 \pm 11.8$ & $4.3 \pm 10.4$ & -7.5 to 0.8 & 0.01 & 0.62 & 8.1 \\
\hline Daytime DBP & $75.5 \pm 8.4$ & $74.2 \pm 8.1$ & $1.3 \pm 5.0$ & -2.9 to 0.3 & NS & 0.82 & 6.6 \\
\hline Daytime HR & $69.1 \pm 8.2$ & $66.9 \pm 8.8$ & $1.1 \pm 8.4$ & -2.9 to 1.3 & NS & 0.79 & 6.7 \\
\hline Nighttime SBP & $118.1 \pm 14.7$ & $118.6 \pm 12.3$ & $-0.5 \pm 13.7$ & -3.5 to 5.2 & NS & 0.49 & 11.6 \\
\hline Nighttime DBP & $67.9 \pm 9.2$ & $68.7 \pm 8.9$ & $-0.8 \pm 7.1$ & -1.2 to 3.2 & NS & 0.70 & 10.3 \\
\hline Nighttime HR & $63.7 \pm 8.2$ & $65.9 \pm 7.9$ & $-1.2 \pm 8.0$ & -1.7 to 0.9 & NS & 0.69 & 8.5 \\
\hline Awake in-bed SBP & $122.3 \pm 14.9$ & $119.4 \pm 14.5$ & $2.9 \pm 12.7$ & $-7.1 \pm 1.2$ & NS & 0.63 & 10.5 \\
\hline Awake in-bed DBP & $69.9 \pm 10.3$ & $68.9 \pm 9.3$ & $1.0 \pm 7.9$ & -1.9 to 3.3 & NS & 0.68 & 11.4 \\
\hline Awake in-bed HR & $64.1 \pm 7.4$ & $64.3 \pm 7.9$ & $1.3 \pm 5.1$ & -0.4 to 2.9 & NS & 0.79 & 6.6 \\
\hline Awake ambulatory SBP & $131.4 \pm 12.4$ & $126.4 \pm 11.4$ & $5.0 \pm 11.2$ & -8.5 to -1.4 & 0.008 & 0.43 & 13.0 \\
\hline Awake ambulatory DBP & $77.0 \pm 9.2$ & $75.3 \pm 8.4$ & $1.7 \pm 5.7$ & $-3.5 \pm 0.1$ & NS & 0.59 & 12.6 \\
\hline Awake ambulatory HR & $69.4 \pm 9.7$ & $67.9 \pm 10.5$ & $-0.7 \pm 5.2$ & -3.3 to 0.4 & NS & 0.55 & 8.2 \\
\hline Asleep SBP & $114.9 \pm 14.9$ & $115.1 \pm 13.3$ & $-0.2 \pm 15.0$ & -4.5 to 5.0 & NS & 0.56 & 8.7 \\
\hline Asleep DBP & $65.3 \pm 9.0$ & $66.0 \pm 9.3$ & $-0.7 \pm 8.3$ & -1.9 to 3.3 & NS & 0.79 & 7.5 \\
\hline Asleep HR & $59.7 \pm 6.4$ & $60.4 \pm 7.5$ & $0.7 \pm 5.2$ & -2.3 to 0.9 & NS & 0.84 & 9.1 \\
\hline Nighttime SBP dip (\%) & $8.9 \pm 7.4$ & $5.2 \pm 4.7$ & $-3.6 \pm 7.1$ & -5.9 to -1.3 & 0.003 & 0.18 & 13.1 \\
\hline Nighttime DBP dip (\%) & $10.1 \pm 7.6$ & $7.4 \pm 5.4$ & $2.7 \pm 8.6$ & -5.4 to 0.06 & NS & 0.18 & 12.5 \\
\hline Nighttime HR dip (\%) & $8.3 \pm 6.5$ & $6.9 \pm 6.6$ & $1.6 \pm 6.6$ & -3.3 to 0.1 & NS & 0.19 & 11.2 \\
\hline
\end{tabular}

Abbreviations: DBP, diastolic blood pressure ( $\mathrm{mm} \mathrm{Hg}$ ); HR, heart rate (beats per minute); NS, not significant; SBP, systolic blood pressure (mm Hg).

ambulatory conditions (Table 2). No differences in awake in-bed, nighttime and asleep SBP, neither in any of the DBP values were found.

During inpatient ABP monitoring, $43 \%$ of the participants reported good, $20 \%$ medium and $38 \%$ bad sleep, compared with 60,20 and $20 \%$, respectively, during outpatient monitoring ( $P=$ not significant). Moreover, during inpatient monitoring, $57 \%$ of the participants reported sleep duration as usual, $8 \%$ as $2 \mathrm{~h}$ less, $18 \%$ as $2-4 \mathrm{~h}$ less and $18 \%>4$ h as less than normal, compared with $65,20,8$ and $8 \%$, respectively, during outpatient monitoring $(P=\mathrm{NS})$.

The nighttime SBP dip (vs. daytime) was larger in the outpatient than in the inpatient setting, whereas the nighttime DBP dip showed a tendency toward increase in the outpatient setting that did not reach statistical significance (Figure 1). In addition, sleeping SBP dip (vs. awake ambulatory and in-bed) was larger in the outpatient setting with no differences in DBP (Figure 1). Sleeping SBP and DBP dips were larger than the nighttime dip in both settings. Specifically, inpatient sleeping and nighttime SBP dips were $7.8 \pm 5.9$ and $5.2 \pm 4.6 \mathrm{~mm} \mathrm{Hg}$, respectively. The DBP was $10.6 \pm 7.1$ (inpatient) $v s$. $7.3 \pm 5.4 \mathrm{~mm} \mathrm{Hg}$ (outpatient). Finally, the outpatient asleep vs. nighttime dip was $11.1 \pm 7.3$ vs. $8.8 \pm 7.5 \mathrm{~mm} \mathrm{Hg}$ for SBP and $13.2 \pm 7.1$ vs. $10.0 \pm 7.6 \mathrm{~mm} \mathrm{Hg}$ for DBP. ( $P<0.05$ for all comparisons.) According to in-hospital ABP monitoring, 7 subjects were classified as dippers, 26 as non-dippers, 7 as reverse dippers and none as extreme dippers, compared with 13,18, 5 and 4, respectively, for outpatient $A B P$ monitoring $(P=0.05)$. The Pearson's correlation coefficients between inpatient and outpatient ABP parameters, the s.d. of the differences and the coefficients of variation are shown in Table 2.

Further, we used differences in 24-h SBP, 24-h DBP, daytime SBP and DBP, and nighttime SBP and DBP as dependent variables, and age, gender, body mass index and antihypertensive medication intake (yes/no) as independent variables in a multiple regression model, which showed that none of the above variables significantly altered the difference between the inpatient and outpatient settings.

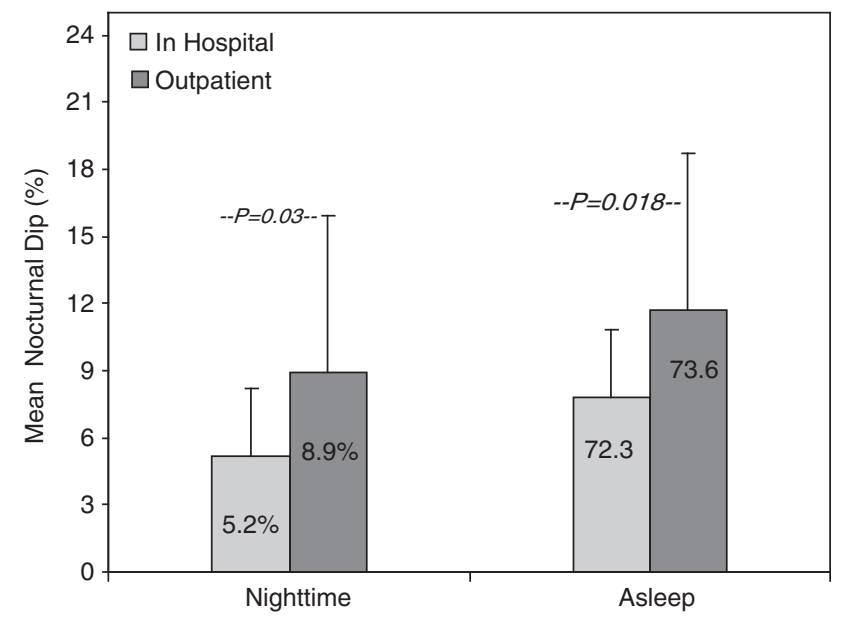

Figure 1 Nighttime and sleeping systolic blood pressure (SBP) dip during inpatient and outpatient ambulatory monitoring.

Bland-Altman plots showing agreement between measurements of daytime SBP and percent of nighttime dipping in the inpatient and outpatient setting can be seen in Figure 2, which shows that both daytime SBP and nocturnal dip were higher in the ambulatory setting than in-hospital.

\section{DISCUSSION}

This study was designed to assess the effect of hospitalization on 24-h ABP in mild-to-moderate hypertension. The main study findings are that, with in-hospital ABP monitoring, (a) daytime SBP is underestimated and (b) the nocturnal SBP dip is underestimated, leading to an overdiagnosis of non-dippers. The dissimilarity between in- and out-of-hospital ABP levels in this study is further supported by the s.d. 

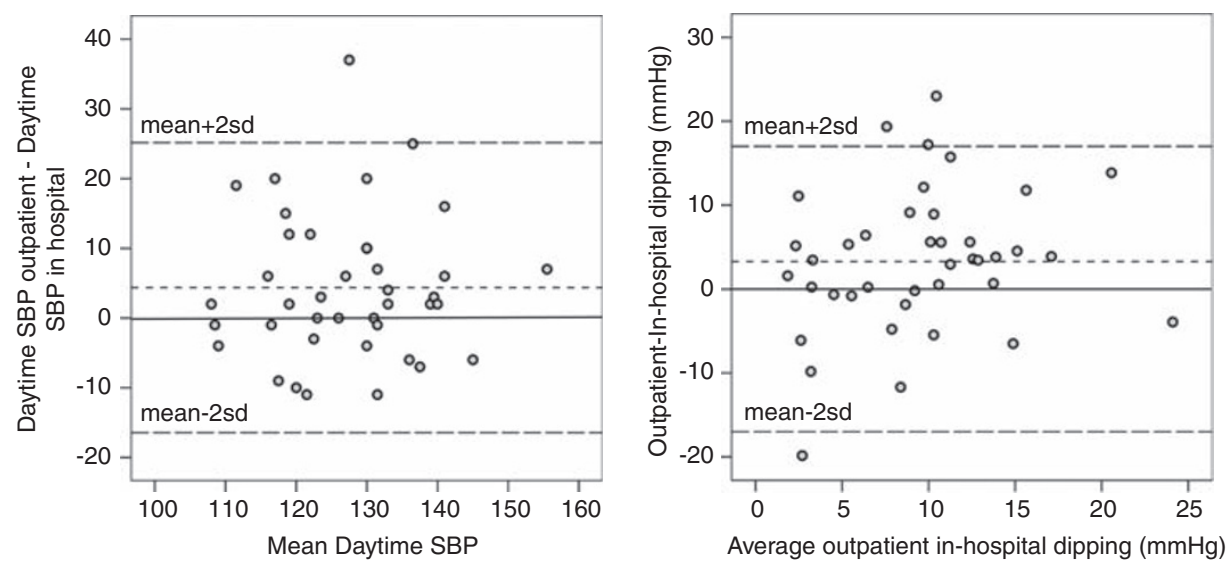

Figure 2 Bland-Altman plots for daytime ambulatory SBP and nocturnal SBP dip (horizontal lines represent mean differences \pm 2 s.d.).

of the differences, which were higher, and the correlation coefficients, which were lower, when compared with previous data regarding the reproducibility of $\mathrm{ABP}$ in untreated hypertensives assessed in routine daily conditions. ${ }^{3}$

There has been relatively less investigation regarding whether 24-h ABP values obtained in-hospital differ from those obtained in the usual daily environment. ${ }^{9,13-16}$ Among five relevant published studies, two showed 24-h ABP to be higher in the outpatient setting, two showed hospital measurements to be higher and one found no effect of hospitalization on 24-h ABP. ${ }^{9,13-16}$ More specifically, an early study that used continuous intra-arterial BP monitoring in nine patients during controlled periods of activity and rest in both inpatient and outpatient settings showed that mean SBP was higher at home (by $14 \mathrm{~mm} \mathrm{Hg}$ ) than in the hospital. ${ }^{9}$ However, the study used an invasive technique and had low power to fully explore the issue of environmental effects on ABP. Similarly, in a study of 44 white-collar workers with untreated mild hypertension, a markedly lower daytime BP was observed on the fourth day of hospitalization compared with a working day (BP $132 / 82$ vs. $138 / 85 \mathrm{~mm} \mathrm{Hg}$, respectively) with no difference in nighttime BP. ${ }^{9,14}$ These results are in accordance with our study even though the mean age of the population was much younger (35 years)..$^{9,14}$

On the other hand, no significant BP differences were detected in a study that compared inpatient and outpatient $\mathrm{ABP}$ in 20 hypertensive and 20 normotensive pregnant women. ${ }^{16}$ However, women receiving antihypertensive medication had a significantly larger difference between home and inpatient BP $(5.8 \mathrm{~mm} \mathrm{Hg})$ compared with the rest of the group $(3.3 \mathrm{~mm} \mathrm{Hg})$. In a multivariate linear regression model, only the use of antihypertensive therapy was associated with larger differences between inpatient and home BP. Furthermore, an analysis of 30 stable heart transplant recipients showed the 24-h ABP to be lower when assessed at home compared with the first day of hospitalization for an annual checkup. ${ }^{15}$ However, these patients do not represent typical hypertensive subjects because they were treated with immunosuppressants (such as cyclosporine, which is known to increase $\mathrm{BP}^{15}$ ) and were subjected to various non-invasive examinations during the inpatient BP measurement.

The most recent attempt to investigate the effect of hospitalization on 24-h ABP was a study of 42 clinically stable patients hospitalized in the departments of internal medicine, visceral surgery and orthopedics at Basel University Hospital in Switzerland. The study found that 24-h DBP, but not SBP, was higher in the hospital than in the outpatient setting. ${ }^{13}$ This difference of $1.8 \mathrm{~mm} \mathrm{Hg}$ was reported as statistically significant $(P=0.016)$, yet cannot be regarded as clinically important. Other disadvantages of the study are that patients were on variable drug treatment plans and were subjected to various diagnostic and therapeutic procedures pertaining to their indication for admission to an internal medicine, surgical or orthopedics department; all of these factors may affect the ABP profile. Psychological factors might also have had a role because a hospital admission may raise concerns about the health of the individual. ${ }^{13}$

Main limitations of the aforementioned studies are that they included highly selected patients, ${ }^{13,14,16}$ that the patients were sometimes treated with drugs known to influence $\mathrm{BP},{ }^{13,16}$ and that various diagnostic and therapeutic procedures were performed that might have also affected BP levels. Furthermore, the sequence of inpatient and outpatient 24-h recordings was not randomized in any of these studies. This omission is important given the fact that Musso et al., ${ }^{17}$ after conducting four ABP measurements, concluded that there was an adaptation from the first to the fourth measurement in normotensive volunteers despite the fact that reproducibility still remained high. Thus, an order-effect bias cannot be excluded in all previous studies that measured the effect of hospitalization on 24-h BP, because they did not randomize the two measurements.

Another limitation of 24-h ABP monitoring in the hospital setting is the in-bed time, considering the fact that during most of the daytime the individual might rest in-bed without sleeping. Resting in-bed during the day at home rather than the hospital environment might be the reason for differences in daytime SBP between the two environments. The present study investigated the SBP differences between awake and sleeping hours on the basis of patients' reported activity diaries. It seems that mean SBP during awake hours is higher in the ambulatory conditions of routine daily life than in the restricted hospital environment. To date, no previous studies have investigated this issue.

Further, an important point addressed in this study is the assessment of differences in the nighttime BP dip and the diagnosis of nondippers in the two settings. The present data showed that normal SBP dipping during nighttime is reduced in the hospital setting, which is mainly attributed to the above-mentioned decline in daytime ABP in the hospital rather than a rise in nocturnal ABP. Although there was a tendency for better and longer sleep at home than in the hospital, these differences neither reached statistical significance nor did they seem to affect average sleeping ABP.

One limitation of our study is the fact that patients had a mild medical condition-namely, open-angle glaucoma-which causes a 
low level of stress/anxiety. The degree of difference in anxiety/stress levels of these patients between the inpatient and outpatient settings could be different from those found with other diseases. However, patients in the present study did not receive any medications, which they were not receiving before admission. Further, they were not in pain. Both these variables could influence BP levels and bias the effect of hospital environment on BP. It should also be noted that despite the higher reproducibility of 24-h BP compared with office measurements, there are some concerns regarding the reproducibility of 24-h BP measurements, which should be taken into account and are a source of bias in any study that uses ABP measurements to assess outcomes. ${ }^{17-20}$

These data suggest that inpatient 24-h ABP monitoring does not reflect the usual BP level during routine daily life or the usual diurnal pattern of an individual. Relying on 24-h ABP monitoring performed in the hospital environment might lead to an underestimation of ABP and overdiagnosis of non-dippers. Therefore, 24-h ABP monitoring for decision making in hypertension should only be applied in the routine daily conditions of each individual.

\section{CONFLICT OF INTEREST}

The authors declare no conflict of interest.

1 Mancia G, De BG, Dominiczak A, Cifkova R, Fagard R, Germano G, Grassi G, Heagerty AM, Kjeldsen SE, Laurent S, Narkiewicz K, Ruilope L, Rynkiewicz A, Schmieder RE, Boudier HA, Zanchetti A, Vahanian A, Camm J, De CR, Dean V, Dickstein K, Filippatos G, Funck-Brentano C, Hellemans I, Kristensen SD, McGregor K, Sechtem U, Silber S, Tendera M, Widimsky P, Zamorano JL, Erdine S, Kiowski W, gabiti-Rosei E, Ambrosioni E, Lindholm LH, Viigimaa M, Adamopoulos S, gabiti-Rosei E, Ambrosioni E, Bertomeu V, Clement D, Erdine S, Farsang C, Gaita D, Lip G, Mallion JM, Manolis AJ, Nilsson PM, O'Brien E, Ponikowski P, Redon J, Ruschitzka F, Tamargo J, van ZP, Waeber B, Williams B. 2007 Guidelines for the management of arterial hypertension: the task force for the management of arterial hypertension of the European Society of Hypertension (ESH) and of the European Society of Cardiology (ESC). J Hypertens 2007; 25: 1105-1187.

2 Chobanian AV, Bakris GL, Black HR, Cushman WC, Green LA, Izzo Jr JL, Jones DW, Materson BJ, Oparil S, Wright Jr JT, Roccella EJ. The seventh report of the joint national committee on prevention, detection, evaluation, and treatment of high blood pressure: the JNC 7 report. Jama 2003; 289: 2560-2572.

3 Stergiou GS, Baibas NM, Kantzarou AP, Skeva II, Kalkana CB, Roussias LG, Mountokalakis TD. Reproducibility of clinic, ambulatory and home blood pressure: implications on the design of trials for the assessment of the efficacy of antihypertensive drugs. J Hypertens 2002; 15: 101-104.
4 Ohkubo T, Imai Y, Tsuji I, Nagai K, Watanabe N, Minami N, Itoh O, Bando T, Sakuma M, Fukao A, Satoh H, Hisamichi S, Abe K. Prediction of mortality by ambulatory blood pressure monitoring versus screening blood pressure measurements: a pilot study in Ohasama. J Hypertens 1997; 15: 357-364.

5 Ohkubo T, Hozawa A, Nagai K, Kikuya M, Tsuji I, Ito S, Satoh H, Hisamichi S, Imai Y. Prediction of stroke by ambulatory blood pressure monitoring versus screening blood pressure measurements in a general population: the Ohasama study. J Hypertens 2000; 18: 847-854.

6 O'Brien E, Asmar R, Beilin L, Imai Y, Mancia G, Mengden T, Myers M, Padfield P, Parati G, Pickering T, Staessen J, Stergiou G, Verdecchia P on behalf of the European Society of Hypertension Working Group on Blood Pressure Monitoring. European society of hypertension recommendations for conventional, ambulatory and home blood pressure measurement. J Hypertens 2003; 21: 821-848.

7 Fotherby MD, Critchley D, Potter JF. Effect of hospitalization on conventional and 24-h blood pressure. Age Ageing 1995; 24: 25-29.

8 Hossmann V, FitzGerald GA, Dollery CT. Influence of hospitalization and placebo therapy on blood pressure and sympathetic function in essential hypertension. Hypertension 1981; 3: 113-118.

9 Young MA, Rowlands DB, Stallard TJ, Watson RD, Littler WA. Effect of environment on blood pressure: home versus hospital. Br Med J (Clin Res Ed) 1983; 286: 1235-1236.

10 Parati G, Mendis S, Abegunde D, Asmar R, Mieke S, Murray A, Shengelia B, Steenvoorden G, Van MG, O'Brien E. Recommendations for blood pressure measuring devices for office/clinic use in low resource settings. Blood Press Monit 2005; 10: 3-10.

11 O'Brien E, Waeber B, Parati G, Staessen J, Myers MG. Blood pressure measuring devices: recommendations of the European Society of Hypertension. BMJ 2001; 322: 531-536.

12 Baumgart P, Kamp J. Accuracy of the SpaceLabs Medical 90217 ambulatory blood pressure monitor. Blood Press Monit 1998; 3: 303-307.

13 Conen D, Martina B, Perruchoud AP, Leimenstoll BM. High prevalence of newly detected hypertension in hospitalized patients: the value of in-hospital 24-h blood pressure measurement. J Hypertens 2006; 24: 301-306.

14 Paschalis-Purtak K, Pucilowska B, Kabat M, Sznajderman M. Clinical evaluation of $24 \mathrm{~h}$ ambulatory monitoring of blood pressure under various environmental conditions (home and work versus hospital). Blood Press Monit 1998; 3: 289-294.

15 Vanhaecke J, Van CJ, Droogne W, Fagard R, Staessen J. Out-patient versus in-hospital ambulatory 24-h blood pressure monitoring in heart transplant recipients. J Hum Hypertens 1999; 13: 199-202.

16 Walker S, Permezel M, Brennecke S, Tuttle L, Ugoni A, Higgins J. The effect of hospitalisation on ambulatory blood pressure in pregnancy. Aust N Z J Obstet Gynaecol 2002; 42: 490-493.

17 Musso NR, Vergassola C, Barone C, Lotti G. Ambulatory blood pressure monitoring: how reproducible is it? Am J Hypertens 1997; 10: 936-939.

18 Cuspidi C, Meani S, Salerno M, Valerio C, Fusi V Severgnini B, Lonati L, Magrini F, Zanchetti A. Reproducibility of nocturnal blood pressure fall in early phases of untreated essential hypertension: a prospective observational study. J Hum Hypertens 2004; 18: 503-509.

19 Eguchi K, Hoshide S, Hoshide Y, Ishikawa S, Shimada K, Kario K. Reproducibility of ambulatory blood pressure in treated and untreated hypertensive patients. $J$ Hypertens 2010; 28: 918-924.

20 Stenehjem AE, Os I. Reproducibility of blood pressure variability, white-coat effect and dipping pattern in untreated, uncomplicated and newly diagnosed essential hypertension. Blood Press 2004; 13: 214-224. 